\title{
Fabrication of Poly(Aspartic Acid)-Nanogold Modified Electrode and Its Application for Simultaneous Determination of Dopamine, Ascorbic Acid, and Uric Acid
}

\author{
Juan Li, Xiaoli Zhang* \\ School of Chemistry and Chemical Engineering, Shandong University, Jinan, China \\ Email: "zhangxl@sdu.edu.cn
}

Received December 22, 2011; revised February 8, 2012; accepted February 24, 2012

\begin{abstract}
A nanostructured polymer film incorporated gold nanoparticles modified electrode was fabricated. The fabrication process involved a previous electropolymerization of aspartic acid and followed by the eletrodeposition of gold nanoparticles on the glassy carbon electrode. The resulting poly(aspartic acid)-nanogold modified electrode (PAAnano-Au/GCE) was characterized by scanning electron microscopy (SEM) and electrochemical impedance spectroscopy (EIS). A higher catalytic activity was obtained to electrocatalytic oxidation of dopamine (DA), ascorbic acid (AA) and uric acid (UA) due to the enhanced peak current and well-defined peak separations compared with three, bare GCE, PAA/GCE and nano-Au/GCE. Simultaneous determination of DA, AA, and UA were studied by voltammetry. The linear range of $5.0 \times 10^{-7}-1.0 \times 10^{-4} \mathrm{M}$ for DA, $5.0 \times 10^{-6}-2.0 \times 10^{-3} \mathrm{M}$ for AA and $5.0 \times 10^{-6}-1.0 \times 10^{-3} \mathrm{M}$ for UA was obtained. The detection limit was calculated for DA, AA and UA as being $6.5 \times 10^{-8} \mathrm{M}, 5.6 \times 10^{-7} \mathrm{M}$ and $3.0 \times$ $10^{-7} \mathrm{M}$, respectively $(\mathrm{S} / \mathrm{N}=3)$. The practical application of the present modified electrode was demonstrated by the determination of DA, AA and UA in calf serum and fetal calf serum samples.
\end{abstract}

Keywords: Simultaneous Determination; Poly(Aspartic Acid)-Nanogold Modified Electrode; Dopamine; Ascorbic Acid; Uric Acid; Voltammetry

\section{Introduction}

Dopamine (DA) is an important neurotransmitter molecule and an indispensable intermediate species in human metabolism [1-3]. The loss of DA may cause some serious diseases, such as schizophrenia and Parkinson's disease [4]. Similarly, ascorbic acid (AA) functions as a redox cofactor and catalyst in a broad array of biochemical reactions and processes. AA is used for the prevention of scurvy and treatment of common cold, mental illness, cancer and AIDS [5]. Uric acid (UA) is produced by xanthine oxidase from xanthine and hypoxanthine, which are, in turn, produced from purine. UA is more toxic to tissues than either xanthine or hypoxanthine because, at high concentrations, it may cause such diseases as hyperuricaemia, gout, and the Lesch-Nyan disease [6]. Usually DA, AA and UA are coexisting in our body fluids. Therefore, it is very important to develop simple and rapid methods for their simultaneous determination in routine analysis.

Electrochemical detection of DA, AA and UA is a preferred method, because they are electrochemically

${ }^{*}$ Corresponding author. active. In addition, electrochemical method offers advantages such as simplicity, speed and sensitivity. A major problem in simultaneous determination is the resolution between DA and AA as well as DA and UA. At nearly all traditional bare electrodes, DA, AA and UA are oxidized at nearly same potential, resulting in an overlapping voltammetric response. To overcome the above problem, a number of studies as reported in the literature have been carried out to develop novel, modified electrodes for simultaneous determination of DA, AA and UA, such as silver doped poly(L-valine) modified GCE [7], zinc oxide composite film [8], palladium nanoparticle-loaded carbon nanofibers modified electrode [9], carbon ceramic electrode prepared by sol-gel technique [10], Pt-Au hybrid film modified electrode [11], carbon ionic liquid electrode [12], and ruthenium oxide modified electrode [13]. In recent years, electro-synthesized polymers modified electrode have been used to detect DA and/or DA, AA and UA, simultaneously, such as poly (vinyl alcohol) [14], poly(evans blue) [15], poly(oracet blue) [16], poly(chromotrope 2B) [17], poly(4-(2-pyridylazo) resorcinol) [18], poly(eriochrome black T) [19], poly(sulfonazo III) [20], poly(N,N'-di- 
methylaniline) [21], poly(3,4-ethylenedioxy) thiophene [22], poly(methylene blue) [23], poly(3,4-ethylenedioxythiopheneco-(5-amino-2-napthalenesulfonic acid)) [24] and poly(5-amino-2-mercapto-1,3,4-thiadiazole) [25] modified electrodes.

On the other hand, nanomaterials show higher electrocatalytic activity, as a kind of special sensing material of chemically modified electrodes. Particularly, the gold nanoparticles have attracted much attention in recent decades, owing to their excellent optical and electronic properties, as well as biocompatible ability. Very recently, we reported the electrodeposition preparation of nanogold modified glassy carbon electrode and its application to simultaneous voltammetric determination of dihydroxyhenzene isomers [26].

Composite electrodes have received great attention in the last decade due to their wide applications in analytical chemistry as chemical and biosensors [27]. Preparation of composite electrodes by incorporation of metal nanoparticles or polymers on the electrode surface can be extensively enhance the catalytic activity of the electrodes towards electrochemical behaviors of many biologically importance molecules $[25,28]$

The poly aspartic acid film has been used to fabricate modified electrode which can enhance the electron transfer rate and reduce the overpotential for the oxidation of AA [29]. Here we report for the first time a simultaneous voltammetric determination of DA, AA and UA using a poly aspartic acid incorporated nanogold particles modified glassy carbon electrode (PAA-nano-Au/GCE). It illustrated that the PAA-nano-Au/GCE not only can catalytically oxidize DA, AA and UA simultaneously, but separate their oxidation peaks distinctly. Compared with the existing reports about simultaneous determination of DA, AA and UA (shown in Table 1), the proposed method is much more convenient to prepare the modified electrode, the selectivity and stability is better.

\section{Experimental Method}

\subsection{Chemicals}

Dopamine was purchased from Sigma-Aldrich. Uric acid, ascorbic acid, $\mathrm{HAuCl}_{4}$ and aspartic acid were obtained from Shanghai Chemical Reagents Co. Ltd. (Shanghai, China). All chemicals were of analytical reagent grade and used without further purification. Phosphate buffer solutions (PBS) with different $\mathrm{pH}$ values were prepared by mixing $0.10 \mathrm{M} \mathrm{Na}_{2} \mathrm{HPO}_{4}$ and $0.10 \mathrm{M} \mathrm{NaH}_{2} \mathrm{PO}_{4}$ and $\mathrm{pH}$ values were adjusted by addition of $1.0 \mathrm{M} \mathrm{H}_{3} \mathrm{PO}_{4}$ and/or $\mathrm{NaOH}$ solution. Freshly prepared solution of DA, $\mathrm{AA}$, and UA were used in all experiments. Doubly distilled water was used throughout the experiments.

\subsection{Apparatus}

A CHI 800 electrochemical analyzer (Shanghai Chenhua Instrument Company, China) was used to perform electrode characterization and voltammetric measurements. A JEOL JSM-7600F Scanning Electron Microscope (SEM) was used to provide the information about the surface topography of electrodes. The Nyquist plots of the EIS measurement were recorded by the Potentiostat/Galvanostat Model 273A. A conventional three-electrode system was employed with a saturated calomel electrode (SCE) as the reference electrode, a platinum wire as the auxiliary electrode and a bare or modified glassy carbon electrode (GCE, $3 \mathrm{~mm}$ in diameter) as the working electrode. All potentials in the text were against SCE. The electrolyte solution was purged with highly purified nitrogen for at least $10 \mathrm{~min}$ before the experiment. The all experiments were sustained in the nitrogen atmosphere and carried out at room temperature.

\subsection{Preparation of the Modified Electrode}

The glassy carbon electrode was polished subsequently with 0.5 and $0.05 \mu \mathrm{m}$ alumina slurry, then thoroughly rinsed with water and sonicated in ethanol and distilled water in turn. After being cleaned, the electrode was continuously $\mathrm{CV}$ scanned from -1.5 to $2.0 \mathrm{~V}$ at $100 \mathrm{mV} \cdot \mathrm{s}^{-1}$ for 30 cycles in $10^{-3} \mathrm{M}$ aspartic acid aqueous solution to form poly aspartic acid film on the electrode surface. Followed, this electrode was taken out and rinsed with water, then was immersed into $0.1 \mathrm{M} \mathrm{KNO}_{3}$ containing $0.4 \mathrm{~g} \cdot \mathrm{L}^{-1} \mathrm{HAuCl}_{4}$, electrodeposition from $\mathrm{HAuCl}_{4}$ was conducted for $60 \mathrm{~s}$ at $-0.2 \mathrm{~V}$. Finally, the resulting electrode, PAA-nano-Au/GCE, was activated by several successive cyclic voltammetric scanning from -0.5 to 1.0 $\mathrm{V}$ with $100 \mathrm{mV} \cdot \mathrm{s}^{-1}$ in phosphate buffer solution ( $\left.\mathrm{pH} 6.5\right)$ until a steady voltammogram was obtained.

\subsection{Analytical Procedure}

The buffer solution was degassed with $\mathrm{N}_{2}$ and kept under a $\mathrm{N}_{2}$ blanket. After quantitative DA, AA and UA were introduced into the electrochemical cell, and then the voltammograms were recorded. The peak current was measured and recorded as a signal $\left(I_{\mathrm{p}}\right)$.

\section{Results and Discussion}

\subsection{Surface Morphology of Poly(Aspartic Acid)-Nanogold Film and Its Electrochemical Properties}

Figure 1 displays the continuous cyclic voltammograms (CVs) of aspartic acid electropolymerization onto a GCE in $10^{-3} \mathrm{M}$ aspartic acid aqueous solution over the potential range of $-1.5 \mathrm{~V}$ to $2.0 \mathrm{~V}$ for 15 cycles at a scan rate 
Table 1. Comparison of working range and detection limit of PAA-nano-Au/GCE with reported methods.

\begin{tabular}{|c|c|c|c|c|c|c|c|c|c|c|}
\hline \multicolumn{3}{|c|}{ Linear range $(\mu \mathrm{M})$} & \multicolumn{3}{|c|}{ Detection limit $(\mu \mathrm{M})$} & \multirow{2}{*}{$\begin{array}{l}\text { Detection } \\
\text { method }\end{array}$} & \multicolumn{3}{|c|}{$\mathrm{E}_{\mathrm{pa}}(\mathrm{V})$} & \multirow{2}{*}{ Reference } \\
\hline DA & AA & UA & DA & AA & UA & & DA & AA & UA & \\
\hline $1-20$ & $25-500$ & $2.5-20$ & 0.11 & 13 & 1.4 & DPV & 0.201 & -0.021 & 0.339 & [1] \\
\hline $6-960$ & $15-240$ & $50-800$ & 0.7 & 1.4 & 4.5 & $\mathrm{CV}$ & 0.184 & 0.20 & 0.313 & [8] \\
\hline $0.5-160$ & $50-4000$ & $2-200$ & 0.2 & 15 & 0.7 & DPV & 0.402 & 0.158 & 0.550 & [9] \\
\hline $0.5-50$ & $5-1000$ & $1-50$ & 0.07 & 1.7 & 0.4 & Amperometry & 0.27 & 0.13 & 0.40 & {$[10]$} \\
\hline $24-384$ & $103-1650$ & $21-336$ & 24 & 103 & 21 & DPV & 0.35 & 0.20 & 0.54 & {$[11]$} \\
\hline $2-70$ & $10-250$ & $2-50$ & 1.4 & 7.6 & 0.6 & DPV & 0.17 & -0.01 & 0.30 & {$[14]$} \\
\hline $\begin{array}{l}0.06-0.80 \\
0.80-8.00\end{array}$ & $40.0-800.0$ & $20.0-600.0$ & 0.02 & 1.3 & 0.4 & DPV & 0.13 & -0.35 & 0.225 & {$[16]$} \\
\hline $20-200$ & $150-1000$ & $10-130$ & 0.02 & 10 & 1.0 & DPV & 0.32 & 0.18 & 0.50 & [19] \\
\hline $75-180$ & $75-187$ & $75-180$ & - & - & - & DPV & 0.34 & 0.17 & 0.48 & {$[30]$} \\
\hline $0.5-100$ & $5-2000$ & $5-1000$ & 0.065 & 0.56 & 0.3 & LSV & 0.2 & 0.0 & 0.35 & This work \\
\hline
\end{tabular}

Abbreviations: differential pulse voltammetry, DPV; linear sweep voltammetry, LSV; cyclic voltammetry, CV.

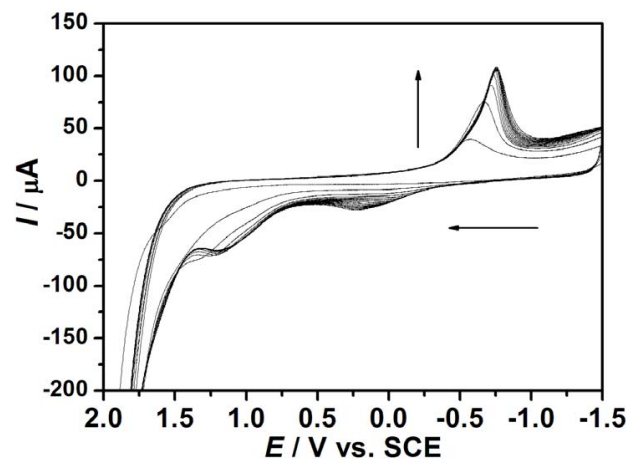

Figure 1. Cyclic voltammograms (CVs) of the poly aspartic acid film growth in the $10^{-3} \mathrm{M}$ aspartic acid aqueous solution. Scan rate $=100 \mathrm{mV} \cdot \mathrm{s}^{-1}$.

of $100 \mathrm{mV} \cdot \mathrm{s}^{-1}$. As can be seen, all the anodic peak and cathodic peak currents enhanced gradually, indicating the formation and growth of an electroactive layer on the GCE surface. After the eighth cycle, the increase of these peaks current tended to be stable, implying that polymerization reach saturation. In addition, a uniform dark blue film presents on the surface of GCE, demonstrating the formation of poly aspartic acid film on GCE. As a primary amine, the electrochemical behavior of aspartic acid is in agreement with the literature [29,31,32]. We demonstrated that the potential range would affect the electropolymerization, only at high potential over $1.5 \mathrm{~V}$, the oxidation of the amino group could turn into its corresponding cation radical, and these cation radicals can form carbon-nitrogen links at the carbon electrode surface. Accordingly, we propose this process illustrated in Scheme 1. The immobilized polymers through electropolymerization have many advantages in the detection of bio-molecules because of its selectivity, sensitivity and homogeneity in electrochemical deposition, strong adherence to electrode surface and chemical stability of the film.

The optimization of the PAA film thickness was also investigated. With the polymerizing cycles increasing, the electroresponse of DA, AA and UA increased at first. But when the polymerizing cycles is more than 15 , the currents begin to fall. This could be explained that an increase in thickness of the film would prevent the electron transfer. Therefore, 15 cycles was used in the experiments.

Figure 2 shows the surface morphologies of bare electrode and modified electrode characterized by scanning electron microscopy (SEM). From the SEM images, It can be seen, the surface of bare GCE is smooth (Figure 2(a)). After separate gold nanoparticles were deposited on GCE, the surface presents spheroidal particles with well-distributed size, and particles' average diameter is about $50 \mathrm{~nm}$ (Figure 2(b)). Similarly, separate poly aspartic acid film on the GCE shows a more uniform surface topography with pycnotic nanostructure with round parvules (Figure 2(c)). After the aspartic acid was previously electropolymerizated on the GCE, the electrodeposition of gold nanoparticles were conducted for $60 \mathrm{~s}$. The small snowflake-like particles could be observed at top level layer. These observations suggest that gold nanoparticles had been incorporated into the initially formed poly aspartic acid film (Figure 2(d)).

The electrochemical impedance spectroscopy (EIS) is an effective method to probe the surface features of the modified electrodes. The Nyquist plot of EIS includes a semicircle portion and a linear portion, with the former at 


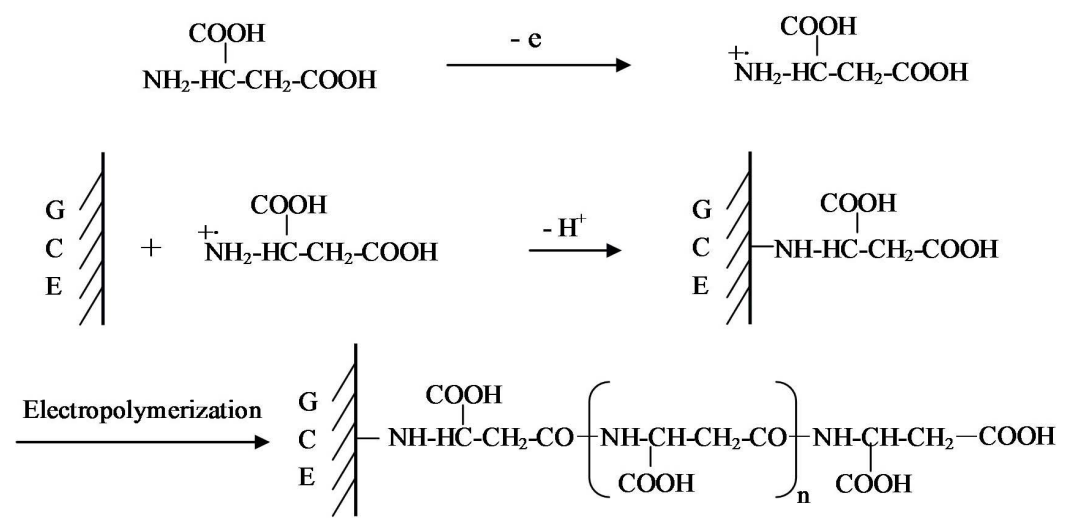

Scheme 1. The modification process of aspartic acid on glassy carbon electrode.
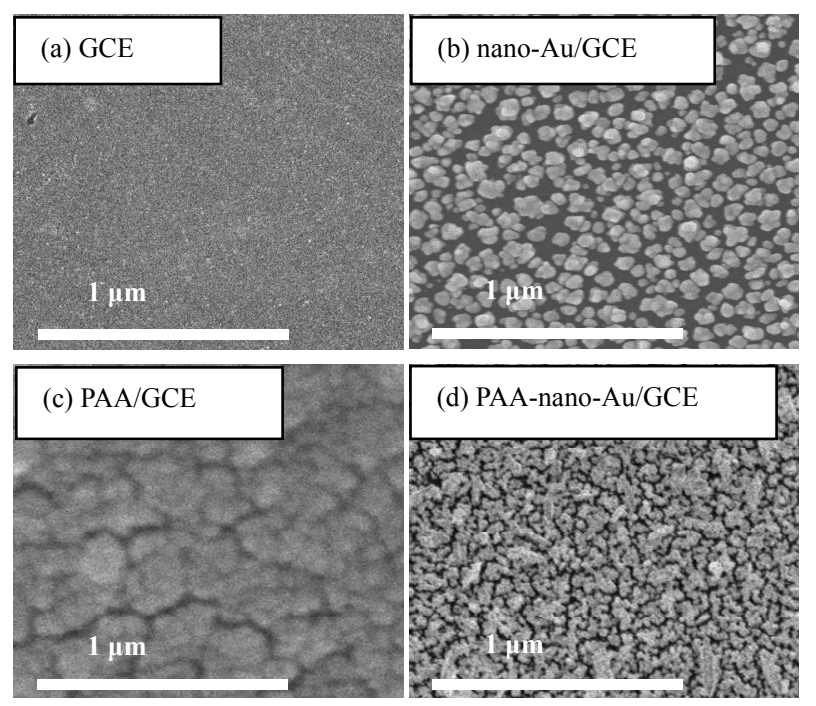

Figure 2. SEM image of (a) Bare GCE; (b) Nano-gold/GCE; (c) Poly Aspartic Acid/GCE; (d) Poly(aspartic acid)-nanogold/ GCE.

higher frequencies corresponding to the electron-transfer limited process and the latter at lower frequencies corresponding to the diffusion process. The electron-transfer resistance $\left(R_{\mathrm{ct}}\right)$ at the electrode surface is equal to the semicircle diameter, which can be used to describe the interface properties of the electrode [33].

Figure 3 displays the EIS of bare GCE (a), PAA/GCE (b), nano-Au/GCE (c) and PAA-nano-Au/GCE (d), respectively, in $0.10 \mathrm{M} \mathrm{KNO}_{3}$ solution containing $5.0 \mathrm{mM}$ $\mathrm{K}_{3} \mathrm{Fe}(\mathrm{CN})_{6} / \mathrm{K}_{4} \mathrm{Fe}(\mathrm{CN})_{6}$ with the frequencies range from 0.1 to $1,00,000 \mathrm{~Hz}$. As shown, the semicircle diameters decrease gradually from Figure 3(a) to (d), which indicates the electron transfer resistance at the electrode/ electrolyte interface decrease gradually. On the other hand, gold nanoparticles can act as electron transfer medium and enhance electron transfer and the result also confirms that the poly aspartic acid film incorporated gold nanoparticles had been successfully fabricated onto the glassy carbon electrode.

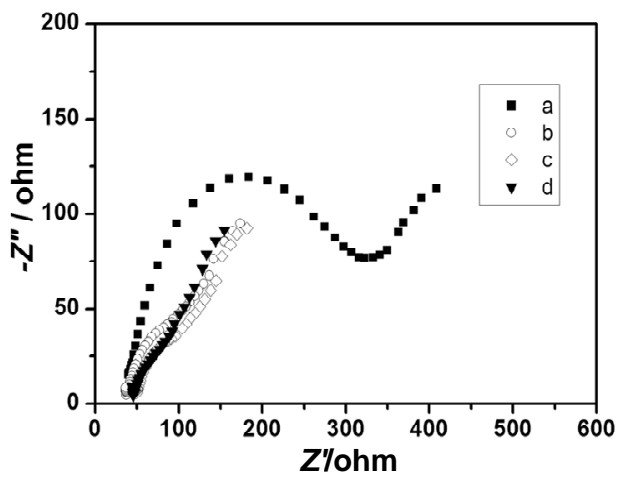

Figure 3. Electrochemical impedance spectroscopy (EIS) of: (a) Bare GCE; (b) Poly aspartic acid/GCE; (c) Nano-gold/ GCE; (d) Poly(aspartic acid)-nanogold/GCE, respectively in

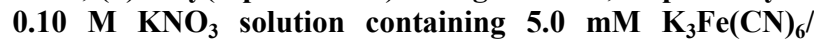
$\mathrm{K}_{4} \mathrm{Fe}(\mathrm{CN})_{6}$.

\subsection{Electrochemical Behavior of $\mathrm{DA}, \mathrm{AA}$ and UA}

The cyclic voltammograms of DA, AA and UA at bare GCE (a), nano-Au/GCE (b), PAA/GCE (c) and PAAnano-Au/GCE (d) in a phosphate buffer solution ( $\mathrm{pH}$ 6.5) containing a ternary mixture of these compounds are shown in Figures 4(A)-(D) are CV recorded on bare GCE (dash line) and PAA-nano-Au/GCE (solid line) for DA, $\mathrm{AA}$ and UA respectively. As can be seen, the oxidation peaks appear at about $0.2 \mathrm{~V}, 0.19 \mathrm{~V}$ and $0.28 \mathrm{~V}$ at bare GCE for DA (B), AA (C) and UA (D), respectively. Bare GCE cannot clearly separate the oxidation peaks of AA and DA in the presence of UA, which showing two indistinguish and broad anodic peaks (Figure 4(A(a))). The phenomenon at nano-Au/GCE is similar to bare GCE (Figure 4(A(b))). Thus, it is unable to determine the three species simultaneously at the bare GCE or separate nano-Au modified electrode.

However, PAA-nano-Au/GCE has significant electrocatalytic activity towards the oxidation reactions of $\mathrm{DA}, \mathrm{AA}$ and UA, and their oxidation peaks move to about $0.2 \mathrm{~V}(\mathrm{~B}), 0.0 \mathrm{~V}(\mathrm{C})$ and $0.35 \mathrm{~V}(\mathrm{D})$, respectively. 


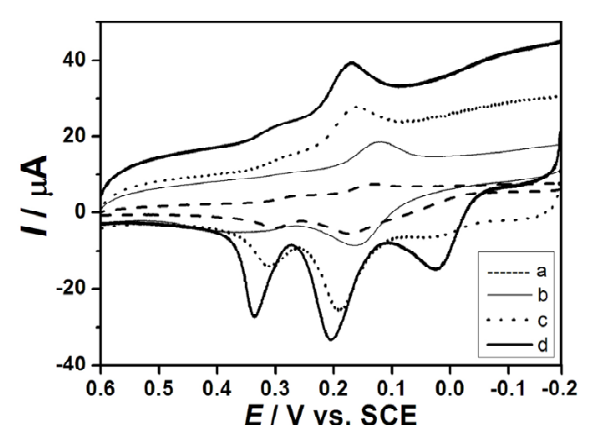

(A)

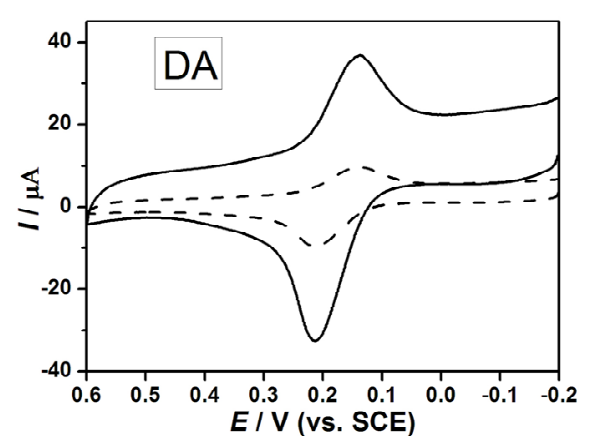

(B)

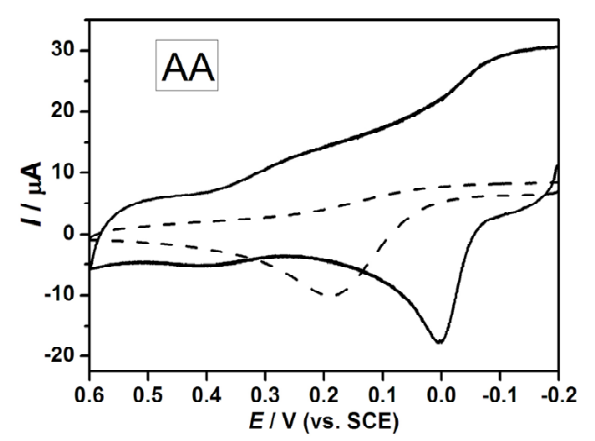

(C)

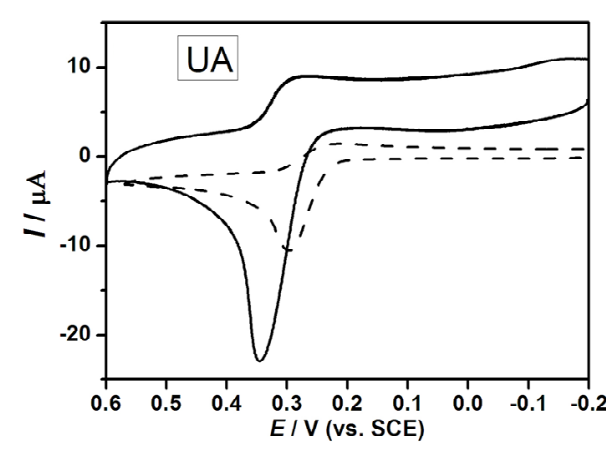

(D)

Figure 4. (A) Cyclic voltammograms of $100.0 \mu M$ UA, 50.0 $\mu M$ DA and 250.0 $\mu \mathrm{M}$ AA at: (a) Bare GCE; (b) Nano-gold/ GCE; (c) Poly aspartic acid/GCE; (d) Poly(aspartic acid)nanogold/GCE in buffer solution $(P h=6.5)$ with scan rate of $100 \mathrm{mV} \cdot \mathrm{s}^{-1}$. (B), (C) and (D) were $\mathrm{CV}$ recordeds on bare GCE (dash line) and PAA-nano-Au/GCE (solid line) for DA, $A A$ and $U A$ respectively.
Also it can be seen that three well-defined oxidation peaks are observed (Figure $\mathbf{4}(\mathbf{A}(\mathrm{d}))$ )), the oxidation peak potential difference $\left(\Delta E_{\mathrm{p}}\right)$ between $\mathrm{AA}$ and $\mathrm{DA}$ is $200 \mathrm{mV}$, $\mathrm{DA}$ and $\mathrm{UA}$ is $150 \mathrm{mV}$, which are large enough separations to allow for the simultaneous determination of DA, AA, and UA in a mixture. Compared with the PAA/GCE (Figure 4(A(c))), the peak currents $\left(I_{\mathrm{P}}\right)$ increased significantly due to the catalytic effect of gold nanoparticles.

In accordance with the literature [29,34], the mechanism for redox process of aspartic acid is a four-electron process. Electrochemical oxidation reactions of DA, AA, and UA at PAA-nano-Au/GCE are depicted in Scheme 2. The obviously enhanced current response and the decrease in the anodic overpotential of about $0.2 \mathrm{~V}$ for AA showed the strong catalytic function of PAA-nano-Au/ GCE to the oxidation of AA. The shift in the overpotentials was due to a kinetics effect, thus a substantial increase in the rate of electron transfer from AA was observed, which was attributed to the improvements in the reversibility of the electron transfer processes $[35,36]$. Because of the effect of both poly aspartic acid film and gold nanoparticles, there were some positive movement in the peak potentials for UA and no obvious change for $\mathrm{DA}$, meanwhile the increased anodic peak currents is striking.

\subsection{Simultaneous Determination of $\mathrm{DA}, \mathrm{AA}$, and UA}

Figure 5 represents the linear sweep voltammograms (LSV) recordings of the solutions containing DA, AA and UA. It can be found that the peak height of each component is proportional to its concentration in the presence of the others. The linear relationships between

DA

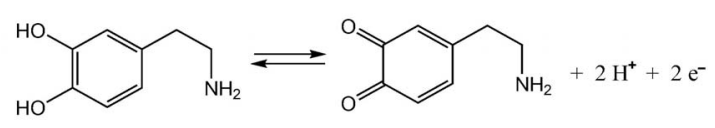

AA

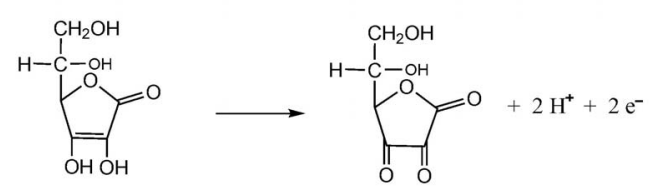

UA
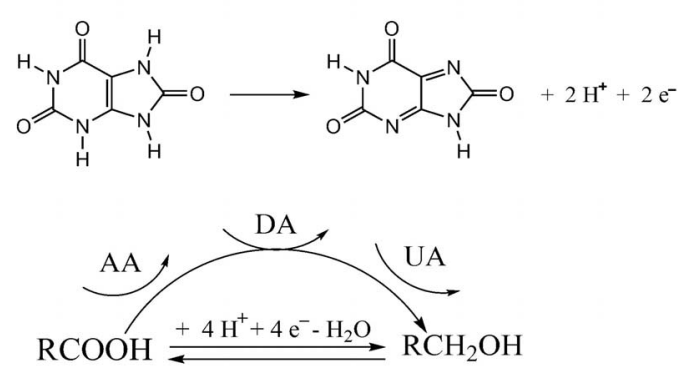

Scheme 2. Electrochemical oxidation reactions of DA, AA, and $U A$ at poly(aspartic acid)-nanogold/GCE. 


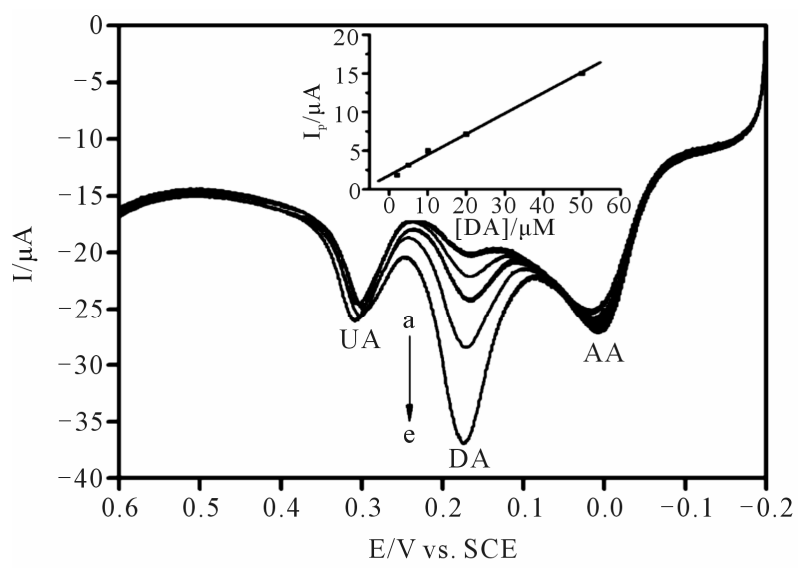

(A)

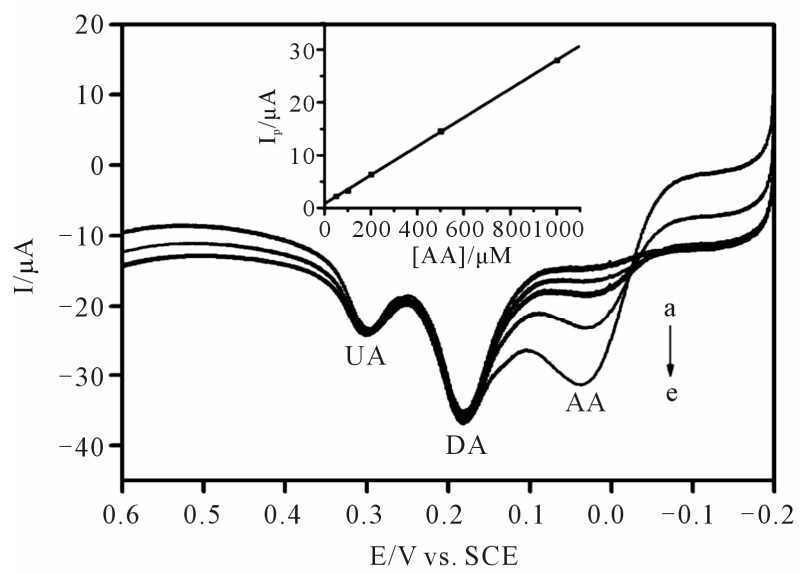

(B)

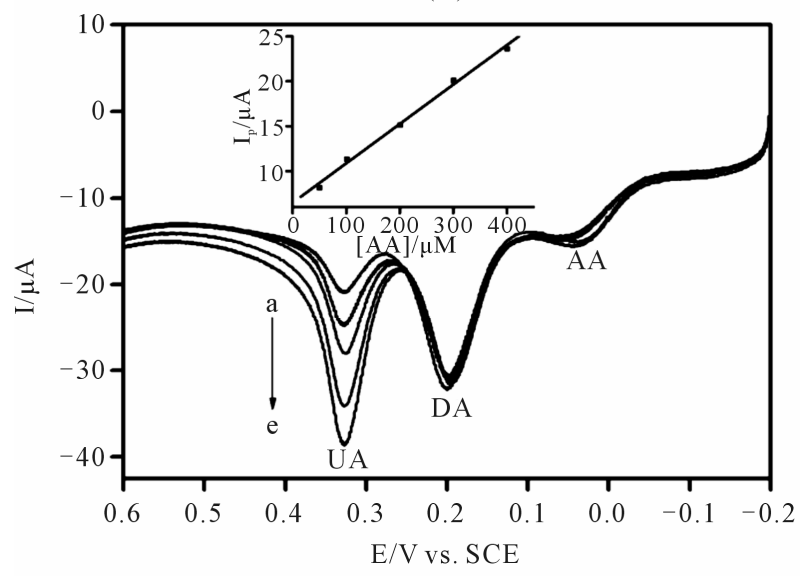

(C)

Figure 5. Voltammograms of (A): LSV of (a) 2.0; (b) 5.0; (c) 10; (d) 20 and (e) $50 \mu \mathrm{M} D$ in the presence of $50.0 \mu \mathrm{M}$ UA and $100.0 \mu \mathrm{M} \mathrm{AA}$; ( insert graph is plot proportionate with these concentrations); (B): (a) 50; (b) 100; (c) 200; (d) 500 and (e) $1000 \mu \mathrm{M} A \mathrm{AA}$ in the presence of $50.0 \mu \mathrm{M} \mathrm{DA}$ and $50.0 \mu \mathrm{M}$ UA; (insert graph is plot proportionate with these concentrations); (C): (a) 50; (b) 100; (c) 200; (d) 300 and (e) $400 \mu \mathrm{M} \mathrm{UA}$ in the presence of $50.0 \mu \mathrm{M} \mathrm{DA}$ and 100.0 $\mu \mathrm{M}$ AA; (insert graph is plot proportionate with these concentrations) at $\mathrm{pH} 6.5$ using the poly(aspartic acid)-nanogold/ GCE with scan rate of $100 \mathrm{mV} \cdot \mathrm{s}^{-1}$. the peak height and the concentration are obtained in the range of $5 \times 10^{-7}-1 \times 10^{-4} \mathrm{M}$ for DA, $5 \times 10^{-6}-2 \times 10^{-3}$ $\mathrm{M}$ for AA and $5 \times 10^{-6}-1 \times 10^{-3} \mathrm{M}$ for $\mathrm{UA}$, respectively. Detection limits of $6.5 \times 10^{-8} \mathrm{M}$ DA, $5.6 \times 10^{-7} \mathrm{M} \mathrm{AA}$ and $3 \times 10^{-7} \mathrm{M}$ UA were obtained (based on $\mathrm{S} / \mathrm{N}=3$ ). These results also confirm that the oxidation processes of DA, AA and UA at the PAA-nano-Au/GCE are independent from each other, which help the simultaneous determination of these three compounds without any interference.

To ascertain the reproducibility of the PAA-nano-Au/ GCE, three same GCE were modified to obtain the PAAnano-Au/GCE, and their responses towards DA, AA and UA were tested. The results indicate that resolution between the voltammetric signals of DA, AA and UA and the sensitivities are the same for all three PAA-nano-Au/ GCE. Besides, the long-term storage stability of PAAnano-Au/GCE was studied by keeping the electrode in $0.1 \mathrm{M}$ phosphate buffer at room temperature. The response of the electrode in the solution containing $5 \times$ $10^{-5} \mathrm{M} \mathrm{DA}, 1 \times 10^{-4} \mathrm{M} \mathrm{AA}$ and $1 \times 10^{-4} \mathrm{M} \mathrm{UA}$, maintained $95 \%, 89 \%, 92 \%$ of the original peak current value after 7 days for DA, AA and UA, respectively. Such a good stability and reproducibility is acceptable for most practical applications.

\subsection{Interference Study}

The possible interferences of some inorganic salts and other organic compounds were also tested with $50.0 \mu \mathrm{M}$ $\mathrm{DA}, 100.0 \mu \mathrm{M}$ AA and $100.0 \mu \mathrm{M}$ UA. The results indicate that five hundred-fold excess (vs DA) of $\mathrm{K}^{+}, \mathrm{Na}^{+}$, $\mathrm{Mg}^{2+}, \mathrm{NO}_{3}^{-}, \mathrm{SO}_{4}^{2-}, \mathrm{Cl}^{-}$and a hundred-fold excess (vs DA) organic compounds such as L-Glutamic acid, tartaric acid, citric acid, glucose, ethanol, and acetone have no influence on the signals of the three compounds with deviations less than $5 \%$.

\subsection{Application to Real Samples}

We examined the applicability of the PAA-nano-Au/ GCE by measuring UA, DA and AA in calf serum and fetal calf serum samples. The calf serum and fetal calf serum samples were diluted five times with phosphate buffer solution before the measurements to prevent the matrix effect of real samples. The results are summarized in Table 2. The recovery of the samples ranged between $92.5 \%$ and $103.8 \%$, indicating the detection procedures are free from interferences of the serum samples matrix.

\section{Conclusions}

The modification of the GCE surface by poly aspartic acid incorporated gold nanoparticles not only improves the electrochemical catalytic activities towards the oxidation 
Table 2. Determination of DA, AA and UA in real samples.

\begin{tabular}{|c|c|c|c|c|c|c|}
\hline & \multicolumn{3}{|c|}{ Sample 1} & \multicolumn{3}{|c|}{ Sample 2} \\
\hline & UA & DA & AA & UA & DA & AA \\
\hline Sample & 26.75 & - & - & 19.99 & - & - \\
\hline Added $\left(10^{-6} \mathrm{M}\right)$ & 100.0 & 50.0 & 500.0 & 100.0 & 50.0 & 500.0 \\
\hline Found $^{[\mathrm{a}]}\left(10^{-6} \mathrm{M}\right)$ & $119.2( \pm 2.1)$ & $51.9( \pm 0.7)$ & $491.8( \pm 4.3)$ & $14.8( \pm 1.7)$ & $51.7( \pm 0.5)$ & $477.5( \pm 4.6)$ \\
\hline Recovery (\%) & 92.5 & 103.8 & 98.4 & 93.1 & 103.4 & 95.5 \\
\hline
\end{tabular}

${ }^{[a]}$ Average values of three measurements.

of DA, AA and UA, but also resolves the overlapped oxidation peaks of DA, AA and UA into three well-defined peaks at potentials 200,0 , and $350 \mathrm{mV}$ in $\mathrm{CV}$, respectively. It also demonstrates that PAA-nano-Au/GCE facilitates the simultaneous determination of DA, AA and UA with good stability, sensitivity and selectivity compared with PAA/GCE or nano-Au/GCE. Due to the electrode's simple fabrication procedure, wide linear range, low detection limit for detection of DA, AA and UA as well as high stability and good reproducibility, this PAAnano-Au/GCE could be applied to the simultaneous determination of $\mathrm{DA}, \mathrm{AA}$ and $\mathrm{UA}$ concentrations in real samples with satisfactory results.

\section{Acknowledgements}

This project was supported by the National Natural Science Foundation of China (Grant No. 20975061), the National Basic Research Program of China (Grant No. 2007CB936602), the Natural Science Foundation of Shandong Province in China (Grant No. Y2008B20).

\section{REFERENCES}

[1] R. P. Da Silva, A. W. O. Lima and S. H. P. Serrano, "Simultaneous Voltammetric Detection of Ascorbic Acid, Dopamine and Uric Acid Using a Pyrolytic Graphite Electrode Modified Into Dopamine Solution," Analytica Chimca Acta, Vol. 612, No. 1, 2008, pp. 89-98. doi:10.1016/j.aca.2008.02.017

[2] X. Zhou, N. Zheng, S. R. Hou, X. J. Li and Z. B. Yuan, "Selective Determination Of Dopamine in the P-Resence of Ascorbic Acid at a Multi-Wall Carbon Nano-TubePoly(3,5-Dihydroxy Benzoic Acid) Film Modified Electrode," Journal of Electroanalytical Chemistry, Vol. 642, No. 1, 2010, pp. 30-34.

doi:10.1016/j.jelechem.2010.01.028

[3] T. J. Yin, W. Z. Wei and J. X. Zeng, "Selective Detection of Dopamine in the Presence of Ascorbic Acid by Use of Glassy-Carbon Electrodes Modified with Both Polyaniline Film and Multi-Walled Carbon Nanotubes with Incorporated $\beta$-Cyclodextrin," Analytical and Bioanalytical Chemistry, Vol. 386, No. 7-8, 2006, pp. 2087-2094. doi:10.1007/s00216-006-0845-Z

[4] J. B. Raoof, A. Kiani, R. Ojani, R. Valiollahi and S. R.
Nadimi, "Simultaneous Voltammetric Determination of Ascorbic Acid and Dopamine at the Surface of Electrodes Modified with Self-Assembled Gold Nanoparticle Films," Journal of Solid State Electrochemistry, Vol. 14, No. 7, 2010, pp. 1171-1176. doi:10.1007/s10008-009-0917-z

[5] O. Arrigoni and M. C. De Tullio, "Ascorbic Acid: More than Just an Antioxidant," Biochimica et Biophysica Acta, Vol. 1569, No. 1-3, 2002, pp. 1-9. doi:10.1016/S0304-4165(01)00235-5

[6] V. V. S. Eswara Dutt and H. A. Mottola, "Determination of Uric Acid at the Microgram Level by a Kinetic Procedure Based on a Pseudo-Induction Period," Analytical Chemistry, Vol. 46, No. 12, 1974, pp. 1777-1781. doi:10.1021/ac60348a041

[7] W. N. Hu, D. M. Sun and W. Ma, "Silver Doped Poly(L-Valine) Modified Glassy Carbon Electrode for the Simultaneous Determination of Uric Acid, Ascorbic Acid and Dopamine," Electroanalysis, Vol. 22, No. 5, 2010, pp. 584-589. doi:10.1002/elan.200900376

[8] C. F. Tang, S. A Kumar and S. M. Chen, "Zinc Oxide/Redox Mediator Composite Films-Based Sensor for Electrochemical Detection of Important Biomolecules," Analytical Biochemistry, Vol. 380, No. 2, 2008, pp. 174183. doi:10.1016/j.ab.2008.06.004

[9] J. S. Huang, Y. Liu, H. Q. Hou and T. Y. You, "Simultaneous Electrochemical Determination of Dopamine, Uric Acid and Ascorbic Acid Using Palladium NanoparticleLoaded Carbon Nanofibers Modified Electrode," Biosensors and Bioelectronics, Vol. 24, No. 4, 2008, pp. 632637. doi:10.1016/j.bios.2008.06.011

[10] A. Salimi, H. MamKhezri and R. Hallaj, "Simultaneous Determination of Ascorbic Acid, Uric Acid and Neurotransmitters with a Carbon Ceramic Electrode Prepared by Sol-Gel Technique," Talanta, Vol. 70, No. 4, 2006, pp. 823-832. doi:10.1016/j.talanta.2006.02.015

[11] S. Thiagarajan and S. M. Chen, "Preparation and Characterization of Ptau Hybrid Film Modified Electrodes and Their Use in Simultaneous Determination of Dopamine, Ascorbic Acid and Uric Acid," Talanta, Vol. 74, No. 2, 2007, pp. 212-222. doi:10.1016/j.talanta.2007.05.049

[12] A. Safavi, N. Maleki, O. Moradlou and F. Tajabadi, "Simultaneous Determination of Dopamine, Ascorbic Acid, and Uric Acid Using Carbon Ionic Liquid Electrode," Analytical Biochemistry, Vol. 359, No. 2, 2006, pp. 224229. doi:10.1016/j.ab.2006.09.008 
[13] P. Shakkthivel and S. M. Chen, "Simultaneous Determination of Ascorbic Acid and Dopamine in the Presence of Uric Acid on Ruthenium Oxide Modified Electrode," Biosensors and Bioelectronics, Vol. 22, No. 8, 2006, pp. 1680-1687. doi:10.1016/i.bios.2006.07.026

[14] Y. X. Li and X. Q. Lin, "Simultaneous Electroanalysis of Dopamine, Ascorbic Acid and Uric Acid By Poly(Vinyl Alcohol) Covalently Modified Glassy Carbon Electrode," Sensors and Actuators B: Chemical, Vol. 115, No. 1, 2006, pp. 134-139. doi:10.1016/j.snb.2005.08.022

[15] L. Q. Lin, J. H. Chen, H. Yao, Y. Z. Chen, Y. J. Zheng and X. H. Lin, "Simultaneous Determination of Dopamine, Ascorbic Acid and Uric Acid at Poly(Evans Blue) Modified Glassy Carbon Electrode," Bioelectrochemistry, Vol. 73, No. 1, 2008, pp. 11-17. doi:10.1016/j.bioelechem.2008.01.009

[16] H. R. Zare, N. Rajabzadeh, N. Nasirizadeh and M. M. Ardakani, "Voltammetric Studies of an Oracet Blue Modified Glassy Carbon Electrode and Its Application for the Simultaneous Determination of Dopamine, Ascorbic Acid and Uric Acid," Journal of Electroanalytical Chemistry, Vol. 589, No. 1, 2006, pp. 60-69. doi:10.1016/j.jelechem.2006.01.011

[17] X. H. Lin, Q. Zhuang, J. H. Chen, S. B. Zhang and Y. J. Zheng, "Electrocatalytic Property of Poly-Chro-Motrope 2B Modified Glassy Carbon Electrode on Dopamine and Its Application," Sensors and Actuators B: Chemical, Vol. 125, No. 1, 2007, pp. 240-245. doi:10.1016/j.snb.2007.02.010

[18] J. H. Chen, J. Zhang, X. H. Lin, H. Y. Wan and S. B. Zhang, "Electrocatalytic Oxidation and Determination of Dopamine in the Presence of Ascorbic Acid and Uric Acid at a Poly (4-(2-Pyridylazo)-Resorcinol) Modified Glassy Carbon Electrode," Electroanalysis, Vol. 19, No. 5, 2007, pp. 612-619. doi:10.1002/elan.200603755

[19] H. Yao, Y. Y. Sun, X. H. Lin, Y. H. Tang and L. Y. Huang, "Electrochemical Characterization of Poly(Eriochrome Black T) Modified Glassy Carbon Electrode and Its Application to Simultaneous Determination of Dopamine, Ascorbic Acid and Uric Acid," Electrochimica Acta, Vol. 52, No. 20, 2007, pp. 6165-6171. doi:10.1016/j.electacta.2007.04.013

[20] A. A. Ensafi, M. Taei, T. Khayamian and A. Arabz-Adeh, "Highly Selective Determination of Ascorbic Acid, Dopamine, and Uric Acid by Differential Pulse Volta-Mmetry Using Poly(Sulfonazo III) Modified Glassy Carbon Electrode," Sensors and Actuators B: Chemical, Vol. 147, No. 1, 2010, pp. 213-221. doi:10.1016/j.snb.2010.02.048

[21] P. R. Roy, T. Okajima and T. Ohsaka, "Simultaneous Electroanalysis of Dopamine and Ascorbic Acid Using Poly(N, N-Dimethylaniline)-Modified Electrodes," Bioelectrochemistry, Vol. 59, No. 1-2, 2003, pp. 11-19. doi:10.1016/S1567-5394(02)00156-1

[22] V. S. Vasantha and S. M. Chen, "Electrocatalysis and Simultaneous Detection of Dopamine and Ascorbic Acid Using Poly(3,4-Ethylenedioxy) Thiophene Film Modified Electrodes," Journal of Electroanalytical Chemistry, Vol. 592, No. 1, 2006, pp. 77-87. doi:10.1016/j.jelechem.2006.04.026
[23] U. Yogeswaran and S. M. Chen, "Multi-Walled Carbon Nanotubes with Poly(Methylene Blue) Composite Film for the Enhancement and Separation of Electroanalytical Responses of Catecholamine and Ascorbic Acid," Sensors and Actuators B: Chemical, Vol. 130, No. 2, 2008, pp. 739-749. doi:10.1016/j.snb.2007.10.040

[24] A. Balamurugan and S. M. Chen, "Poly(3,4-Ethyl-enedioxythiophene-co-(5-amino-2-naphthalenesulfonic acid)) (PEDOT-PANS) Film Modified Glassy Carbon Electrode for Selective Detection of Dopamine in the Presence of Ascorbic Acid and Uric Acid," Analytica Chimica Acta, Vol. 596, No. 1, 2007, pp. 92-98. doi:10.1016/j.aca.2007.05.064

[25] P. Kalimuthu and S. A. John, "Electropolymerized Film of Functionalized Thiadiazole on Glassy Carbon Electrode for the Simultaneous Determination of Ascorbic Acid, Dopamine and Uric Acid," Bioelectrochemistry, Vol. 77, No. 1, 2009, pp. 13-18. doi:10.1016/j.bioelechem.2009.04.010

[26] L. Han and X. L. Zhang, "Simultaneous Voltammetry Determination of Dihydroxybenzene Isomers by Nanogold Modified Electrode," Electroanalysis, Vol. 21, No. 2, 2009, pp. 124-129. doi:10.1002/elan.200804403

[27] B. Serra, S. Jiménez, M. L. Mena, A. J. Reviejo and J. M. Pingarrón, "Composite Electrochemical Biosensors: A Comparison of Three Different Electrode Matrices for the Construction of Amperometric Tyrosinase Biosensors," Biosensors and Bioelectronics, Vol. 17, No. 3, 2002, pp. 217-226. doi:10.1016/S0956-5663(01)00269-X

[28] P. Santhosh, A. Gopalan and K. P. Lee, "Gold Nanoparticles Dispersed Polyaniline Grafted Multiwall Carbon Nanotubes as Newer Electrocatalysts; Preparation and Performances for Methanol Oxidation," Journal of $\mathrm{Ca}$ talysis, Vol. 238, No. 1, 2006, pp. 177-185. doi:10.1016/j.jcat.2005.12.014

[29] L. Zhang and X. Q. Lin, "Electrochemical Behavior of a Covalently Modified Glassy Carbon Electrode with Aspartic Acid and Its Use for Voltammetric Differentiation of Dopamine and Ascorbic Acid," Analytical and Bioanalytical Chemistry, Vol. 382, No. 7, 2005, pp. 1669 1677. doi:10.1007/s00216-005-3318-x

[30] U. Yogeswaran and S. M. Chen, "Separation and Concentration Effect of $f$-MWCNTs on Electrocatalytic Responses of Ascorbic Acid, Dopamine and Uric Acid at $f$-MWCNTs Incorporated with Poly(Neutral Red) Composite Films," Electrochimica Acta, Vol. 52, No. 19, 2007, pp. 5985-5996. doi:10.1016/j.electacta.2007.03.047

[31] A. J. Downard and A. B. Mohamed, "Suppression of Protein Adsorption at Glassy Carbon Electrodes Covalently Modified with Tetraethylene Glycol Diamine," Electroanalysis, Vol. 11, No. 6, 1999, pp. 418-423. doi:10.1002/(SICI)1521-4109(199905)11:6<418::AID-E LAN418>3.0.CO;2-B

[32] R. S. Deinhammer, H. Mankit, J. W. Anderegg and M. D. Porter, "Electrochemical Oxidation of Amine-Containing Compounds: A Route to the Surface Modification of Glassy Carbon Electrodes," Langmuir, Vol. 10, No. 4, 1994, pp. 1306-1313. doi:10.1021/la00016a054

[33] G. Zhao, J. J. Xu and H. Y. Chen, "Fabrication, Charac- 
terization of $\mathrm{Fe}_{3} \mathrm{O}_{4}$ Multilayer Film and Its Application in Promoting Direct Electron Transfer of Hemoglobin," Electrochemistry Communication, Vol. 8, No. 1, 2006, pp. 148-154. doi:10.1016/j.elecom.2005.11.001

[34] H. X. Luo, Z. J. Shi, N. Q. Li, Z. N. Gu and Q. K. Zhuang, "Investigation of the Electrochemical and Electrocatalytic Behavior of Single-Wall Carbon Nanotube Film on a Glassy Carbon Electrode," Analytical Chemistry, Vol. 73, No. 5, 2001, pp. 915-920. doi:10.1021/ac0009671

[35] F. Malem and D. Mandler, "Self-Assembled Monolayers in Electroanalytical Chemistry: Application of Omegamer- capto Carboxylic Acid Monolayers for the Electrochemical Detection of Dopamine in the Presence of a High Concentration of Ascorbic Acid," Analytical Chemistry, Vol. 65, No. 1, 1993, pp. 37-41. doi: $10.1021 / \mathrm{ac} 00049 \mathrm{a} 009$

[36] L. Zhang and X. Q. Lin, "Covalent Modification of Glassy Carbon Electrode with Glutamic Acid for Simultaneous Determination of Uric Acid and Ascorbic Acid," Analyst, Vol. 126, No. 3, 2001, pp. 367-370.

doi:10.1039/b009415n 